\title{
Unraveling the Crystal Structure of All-Inorganic Halide Perovskites using CBED and Electron Ptychography
}

Roberto dos Reis ${ }^{1}$, Hao Yang ${ }^{1}$, Colin Ophus ${ }^{1}$, Tetiana Shalapska ${ }^{2}$, Gregory Bizarri ${ }^{2}$, Didier Perrodin ${ }^{2}$, Peter Ercius ${ }^{1}$, Jim Ciston ${ }^{1}$, Edith Bourret ${ }^{2}$ and Ulrich Dahmen ${ }^{1}$.

${ }^{1 .}$ National Center for Electron Microscopy, Molecular Foundry, LBNL, CA, USA

2. Materials Sciences Division, LBNL, Berkeley, CA, USA.

The unique electronic properties observed in halide perovskites originate in their rich structural complexity that allows compatibility with a variety of structural motifs and compounds. Adjustments of the corner-connected $\mathrm{BX}_{6}$ octahedral network in the $\mathrm{ABX}_{3}$ structure promote a wide range of optical and electronic properties [1]. For this reason, methods to precisely identify the local symmetry are necessary to unambiguously distinguish different possible structural phases. Here, we determine the crystallography of all-inorganic $\mathrm{CsPbBr}_{3-\mathrm{x}} \mathrm{Cl}_{\mathrm{x}}$ perovskite single crystals, grown via the BridgmanStockbarger method, by exploring information contained in electron diffraction patterns. Due to the material's sensitivity to the electron beam, precise atomistic studies of halide perovskites by transmission electron microscopy (TEM), scanning TEM (STEM) and electron diffraction are relatively underdeveloped. Consequently, X-ray diffraction has been the technique of choice to assign crystal structures, requiring a careful study of the splitting of certain lines and of the presence of superlattice reflections. Effects due to small atomic shifts can easily be missed in X-ray diffraction, which accounts for reports of conflicting structures.

Until now, there appears to be no definitive conclusion about the stable structure of these materials as it seems to be largely dependent on the conditions and methods of growth. The possible structures can be narrowed down by following the pathways of distortions from the ideal cubic structure following the group-subgroup relationship shown in Fig. 1a. We use convergent-beam electron diffraction (CBED) acquired at low-temperature $(\sim 70 \mathrm{~K})$ to identify the symmetry of $\mathrm{CsPbBr}_{3-\mathrm{x}} \mathrm{Cl}_{\mathrm{x}}$ single crystals. Highsymmetry CBED pattern containing information on high-order Laue zones (HOLZ), in which the intensities of certain reflections are quite sensitive to small rotations of the octahedral unit, reveal the whole crystal symmetry and positions of $\mathrm{Br} / \mathrm{Cl}$ anions. Figure $1 \mathrm{~b}$ presents $\mathrm{CBED}$ pattern taken along the pseudocubic [001] axis from $\mathrm{CsPBr}_{3}$ sample $(\mathrm{x}=0)$ showing HOLZ reflections. This high-symmetry axis, $\left([001]_{\mathrm{pc}}\right)$ is perpendicular to the alternating layers where the displacements of $\mathrm{Br}$ (or $\mathrm{Cl}$ ) take place during phase transformations from cubic-to-tetragonal-to-orthorhombic. The pattern clearly exhibits a fourfold rotation and two mirror planes, indicated by the arrowheads $m 1$ and $m 2$ and the dotted semicircle in Figure 1b, with the whole-pattern symmetry denoted as $4 \mathrm{~mm}$.

Local imaging of the projected atomistic distribution in these crystals is achieved by simultaneous atomic-resolution electron ptychography (a dose efficient diffractive imaging technique [2]) and Zcontrast, using a Gatan K2-IS direct electron detector. A relatively low dose $\left(10^{3} \mathrm{e} / \AA^{2}\right)$ is employed to acquire 4D-STEM datasets [3] of $256 \times 256 \times 1920 \times 895$ at 800 frames/s with the sample kept at room temperature. The probe-forming aperture is about $17.2 \mathrm{mrad}$. Figure $2 \mathrm{a}$ shows the simultaneously acquired ADF image alongside the ptychographically reconstructed amplitude (Fig. 2b) and phase (Fig. 2c) taken from the same $\mathrm{CsPBr}_{3}$ sample, using the Wigner distribution deconvolution (WDD) approach $[2,4]$. Combination of both techniques, HOLZ-CBED and ptychography, depicts a tetragonal crystal system I4/mcm to $\mathrm{CsPbBr}_{3}$ and $\mathrm{CsPb}(\mathrm{BrCl})_{3}$ whereas $\mathrm{CsPbCl}_{3}$ is found to be orthorhombic [5]. 
References:

[1] M. R. Filip et al, Nat. Commun. 5 (2014), p. 5757.

[2] H. Yang et al, Nat. Commun. 7 (2016), p. 12532.

[3] C. Ophus et al, Microsc. Microanal. 20 (S3) (2014), p. 62.

[4] Rodenburg et al, Philos. Trans. R. Soc. A Math. Phys. Eng. Sci. 339 (1992), p. 521.

[5] Electron microscopy was performed at the National Center for Electron Microscopy/Molecular

Foundry, LBNL, supported by the Office of Science, Office of Basic Energy Sciences, US Department of Energy (DE-AC02-05CH11231). This work was supported by the U.S. Department of Homeland Security/DNDO and was carried out at the Lawrence Berkeley National Laboratory under Contract no. DE-AC02-05CH11231.

a

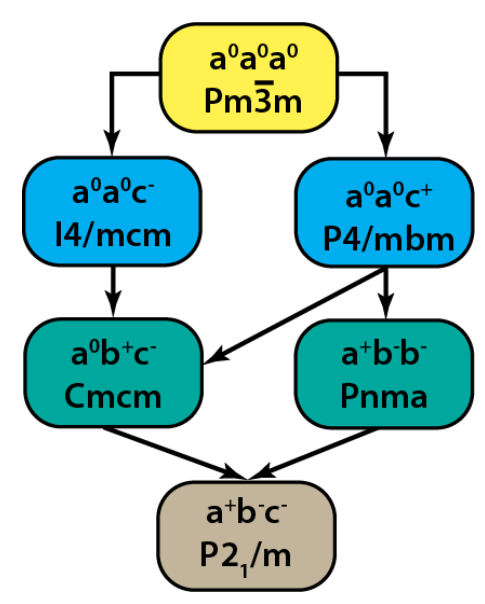

b

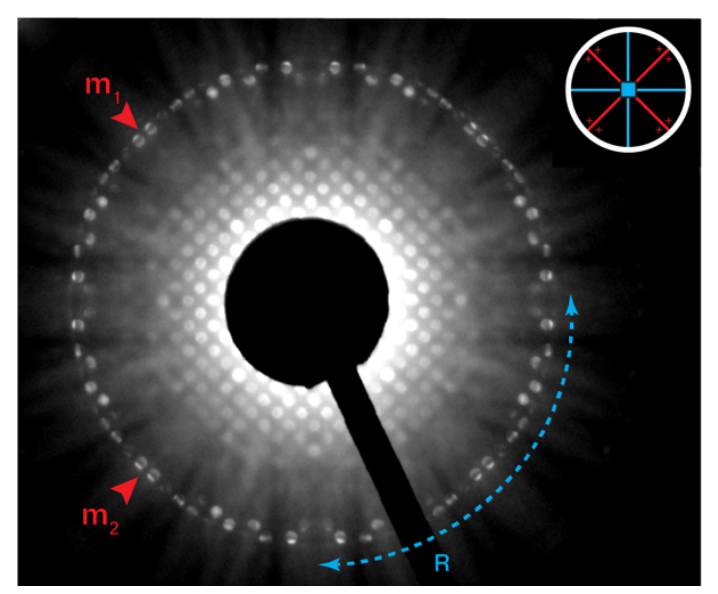

Figure 1. (a) Group-subgroup relationship between the space groups observed in $\mathrm{CsPbBr}_{3-\mathrm{x}} \mathrm{Cl}_{\mathrm{x}}$. The tilt systems are given using Glazer's notation. (b) Low-temperature high-symmetry CBED pattern along $[001]_{\mathrm{pc}}$ axis with the total projected symmetry $4 \mathrm{~mm}$ (symbol shown as inset). Arrowheads $m 1$ and $m 2$ highlight the two mirror symmetries and the dotted semi-circle indicates four-fold rotation.

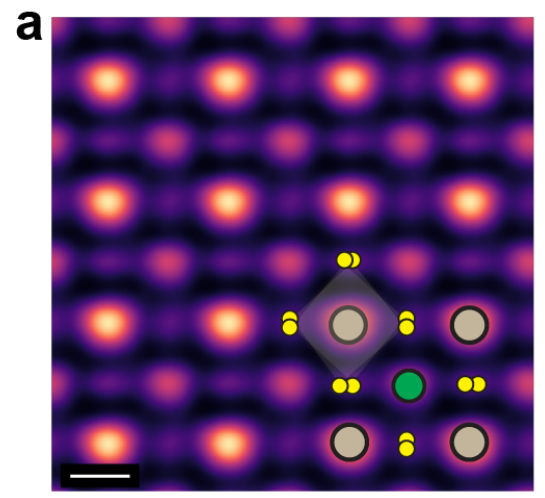

Figure 2. Simultaneous ADF (a) and ptychographically reconstructed amplitude (b) and phase (c) images of $\mathrm{CsPbBr}_{3}$. A clear enhancement of $\mathrm{Br}$ sites is observed in the phase image. The projected atomic positions are marked in the ADF image: $\mathrm{Cs}$ - green, $\mathrm{Pb}$ - gray and $\mathrm{Br}$ - yellow. The bar scale in (a) corresponds to $3 \AA$. 